ARTICLE

\title{
Overtemperature-protection intelligent molecular chiroptical photoswitches
}

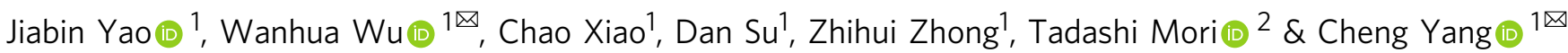

Stimuli-responsive intelligent molecular machines/devices are of current research interest due to their potential application in minimized devices. Constructing molecular machines/ devices capable of accomplishing complex missions is challenging, demanding coalescence of various functions into one molecule. Here we report the construction of intelligent molecular chiroptical photoswitches based on azobenzene-fused bicyclic pillar[ $n]$ arene derivatives, which we defined as molecular universal joints (MUJs). The Z/E photoisomerization of the azobenzene moiety of MUJs induces rolling in/out conformational switching of the azobenzene-bearing side-ring and consequently leads to planar chirality switching of MUJs. Meanwhile, temperature variation was demonstrated to also cause conformational/chiroptical inversion due to the significant entropy change during the ring-flipping. As a result, photo-induced chiroptical switching could be prohibited when the temperature exceeded an upper limit, demonstrating an intelligent molecular photoswitch having over-temperature protection function, which is in stark contrast to the low-temperature-gating effect commonly encountered.

\footnotetext{
${ }^{1}$ Key Laboratory of Green Chemistry \& Technology of Ministry of Education, College of Chemistry, State Key Laboratory of Biotherapy, West China Medical Center, and Healthy Food Evaluation Research Center, Sichuan University, Chengdu, China. ${ }^{2}$ Department of Applied Chemistry, Osaka University, Suita, Japan.

凶email:wuwanhua@scu.edu.cn; yangchengyc@scu.edu.cn
} 
$\mathrm{M}$ olecular machines/devices are miniaturized artificial devices, down to nano- to sub-nanoscales, which can accomplish large-amplitude mechanical motions by responding to input stimuli ${ }^{1-3}$. By virtue of an in-depth understanding of molecular motional physics, sophisticated design and organic synthesis, and stimulus-responsive molecular transformations, molecular machines/devices with complex structures and functions have been constructed ${ }^{4}$, such as molecular shuttles $^{5-9}$, motors ${ }^{10-13}$, pumps $s^{14-16}$, and elevators ${ }^{17}$. These molecular machines/devices can switch among multi-isomeric states ${ }^{18-20}$ by specifically responding to external stimuli, such as solvents ${ }^{21,22}$, light ${ }^{23-26}, \mathrm{pH}^{27,28}, \operatorname{redox}^{29-31}$, and chemical additives $^{32,33}$. Temperature is a universal environmental factor. Unlike the above external stimuli, exerting a thermal effect on a molecular device without affecting its surrounding molecules is difficult. Lowering the temperature could lead to inhibition of some molecular physicochemical pathways, a phenomenon called low-temperature gating ${ }^{34}$. Increasing the temperature generally leads to improved reactivity and conversion pathways, and hightemperature gating has never been observed at the singlemolecule level. Therefore, realizing overtemperature protection, which is vital for switching devices/power transistors, such as the stable operation of computers and devices and safety of power supplies, with a molecular device is obviously challenging. Herein, we report an overtemperature-protection function by integrating the thermo- and photoresponsive functions into one pillar $[n]$ arene-based bicyclic pseudocatenanes, so-called molecular universal joints (MUJs) ${ }^{30,35}$.

\section{Results}

Azobenzene-bearing MUJ1 and MUJ2 (Fig. 1a) were synthesized by etherification of a triethylene glycol ether-modified transazobenzene derivative with dihydroxylated pillar[6]arene ( $\mathrm{P}[6])$ and pillar[5]arene $(\mathrm{P}[5])$ derivatives, respectively, while MUJ3 was obtained by etherification of the tetraethylene glycol ethermodified trans-azobenzene derivative with a dihydroxylated P[6] derivative ${ }^{30}$. The chemical structure of the MUJs was characterized by HR-mass spectrometry and NMR spectroscopic studies. As shown in Fig. 1b, the self-included "in" and self-excluded "out" conformers of the trans-MUJ enantiomers can interconvert quickly accompanied by the switching of chiral conformers that have clockwise-directed $\left(R_{\mathrm{p}}\right.$ conformer $)$ and anticlockwisedirected $\left(S_{\mathrm{p}}\right.$ conformer $)$ arrangement of the substituents, respectively ${ }^{36,37}$. However, for cis-MUJ, the large steric hindrance of cis-azobenzene prevents the self-inclusion by the cavity of pillar [6]arene and inhibits the in-out interconversion.

The azobenzene moiety of the MUJs readily underwent cis/trans photoisomerization upon wavelength-selective photoirradiation. trans-MUJ1 showed strong absorption peaks at $294 \mathrm{~nm}(\varepsilon=$ $\left.25,896 \mathrm{~cm}^{-1} \mathrm{M}^{-1}\right)$ and $365 \mathrm{~nm}\left(\varepsilon=17,093 \mathrm{~cm}^{-1} \mathrm{M}^{-1}\right)$, assignable to the transitions of the $\mathrm{P}[6]$ and trans-azobenzene moieties, respectively. Photoirradiation at $365 \mathrm{~nm}$ led to a rapid decrease in the absorption band at $365 \mathrm{~nm}$ (Fig. 2a) at which the ratio of the absorption spectrum of trans-isomer divided by that of cis-isomer reaches the maximum, accompanied by an increase in the longer wavelength peak at $450 \mathrm{~nm}$ due to the transformation to cis-azobenzene. The ${ }^{1} \mathrm{H}-\mathrm{NMR}$ spectral studies indicated that irradiation with a $365 \mathrm{~nm}$ LED lamp for 2 min led to a significant upfield shift of proton $a$ of the azobenzene moiety (Fig. 2b). Meanwhile, proton $b$ of the side ring-bearing hydroquinone unit showed a downfield shift while upfield shift could be observed with protons of several other hydroquinone units (Fig. 2b), suggesting a significant conformational change from self-included in to self-excluded out conformation upon the trans to cis isomerization, which released the shielding and deshielding effects unequivently exerted on pillar [6]arene subunits. trans-MUJ1 was almost completely converted into cis-MUJ1 in the photostationary state (PSS) under $365 \mathrm{~nm}$ photoirradiation (Fig. 3b). This was also confirmed by HPLC analyses, which gave a cis/trans ratio of 97.8/2.2 in the PSS under $365 \mathrm{~nm}$ irradiation (Supplementary Fig. 38). High cis/trans ratios (>93.5/6.5) were also observed with MUJ2 and MUJ3 (Supplementary Table 1) due to the overwhelmingly high absorption of the trans-MUJs at $365 \mathrm{~nm}$. The lifetime of cis isomers of MUJs will be discussed later.

The spectrum of trans-MUJ1 was almost entirely recovered under photoirradiation of cis-MUJ1 at $510 \mathrm{~nm}$ (Fig. 2a, Supplementary Fig. 38). The trans/cis ratios were in the range of $4.4 \sim 12.0$ in the PSS at $510 \mathrm{~nm}$. However, the ${ }^{1} \mathrm{H}-\mathrm{NMR}$ spectra of cis-MUJ1 ultimately returned to that of trans-MUJ1 after being kept in the dark for one week (Fig. 2b), suggesting that transMUJ1 is a thermodynamically stable species ${ }^{38}$. Detailed HPLC traces suggested that the complete conversion of cis-MUJ1 to trans-MUJ1 in the dark required more than $66 \mathrm{~h}$ at room temperature (Supplementary Fig. 41). In contrast, photoirradiation of cis-MUJ1 at $510 \mathrm{~nm}$ led to the PSS within several minutes. ${ }^{1} \mathrm{H}$ NMR investigation of the photoisomerization between the transand cis-MUJs demonstrated that the photoisomerization is reversible and repeatable without destroying the chemical structure of the MUJs (Supplementary Figs. 80-82). (a)

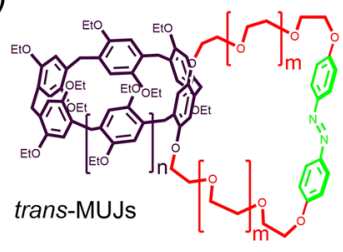

\begin{tabular}{lll}
\hline & $\mathrm{n}$ & $\mathrm{m}$ \\
\hline MUJ1 & 1 & 1 \\
MUJ2 & 0 & 1 \\
MUJ3 & 1 & 2 \\
\hline
\end{tabular}

(b)
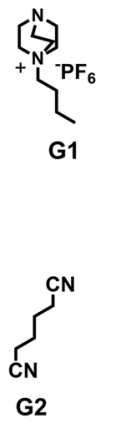

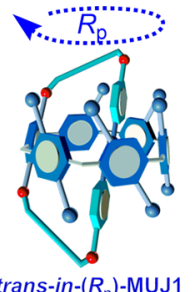

trans-in- $\left(R_{\mathrm{p}}\right)-\mathrm{MUJ1}$

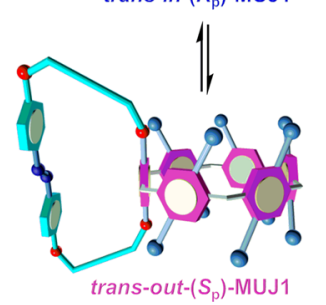

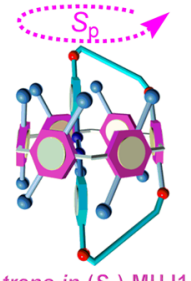

trans-in- $\left(S_{\mathrm{p}}\right)-\mathrm{MUJ1}$

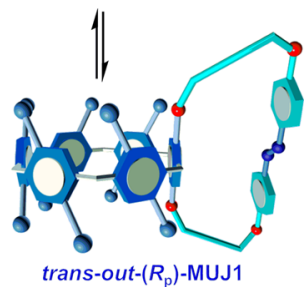

Fig. 1 Chemical structures of MUJs and the in-out conformational switching of trans-MUJs. a Chemical structures of MUJ1, MUJ2, MUJ3, G1, and G2 b Schematic diagram for the in-out equilibrium of the enantiomers of trans-MUJ1. 
(a)
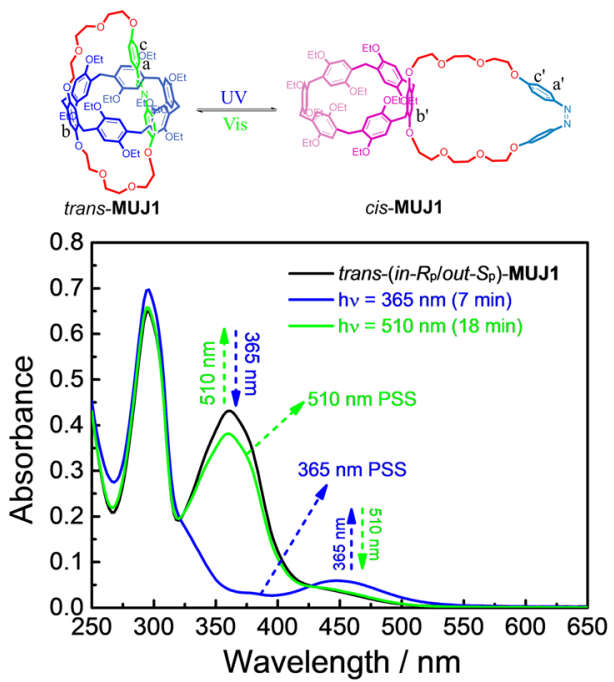

(b)

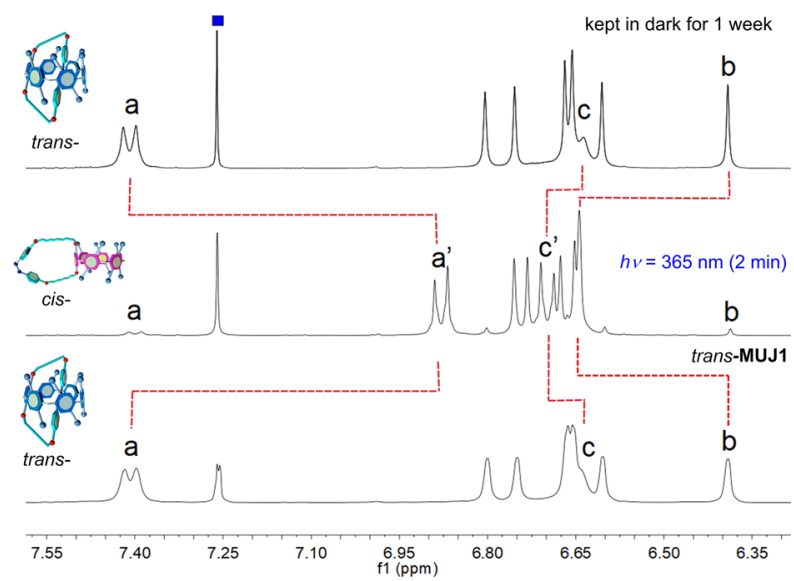

Fig. 2 Photoisomerization of MUJ1 by wavelength-selective photoirradiation. a UV-vis absorption spectra of trans-MUJ1 ( $0.025 \mathrm{mM}$, chloroform) by alternating irradiation at 365 and $510 \mathrm{~nm}$ (xenon grating spectrometer). b ${ }^{1} \mathrm{H}$ NMR spectra of $\mathbf{M U J 1}\left(2 \mathrm{mM}, 400 \mathrm{MHz}_{1} \mathrm{rt}\right)$ in $\mathrm{CDCl} 3 \mathrm{before}($ bottom) and after (middle) irradiation at $365 \mathrm{~nm}$ for $2 \mathrm{~min}$ (LED illuminant) and then after (top) being kept in the dark for 1 week.

(a)

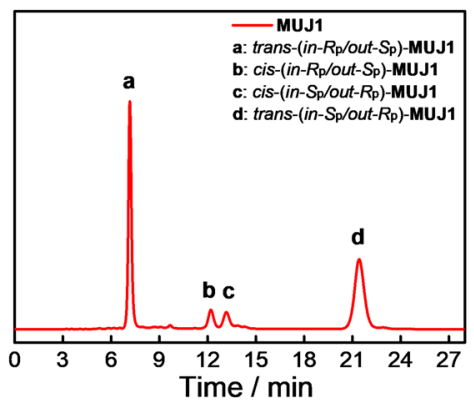

(b)

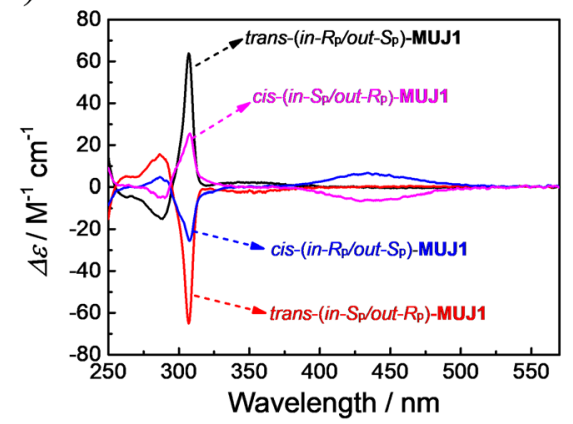

(c)

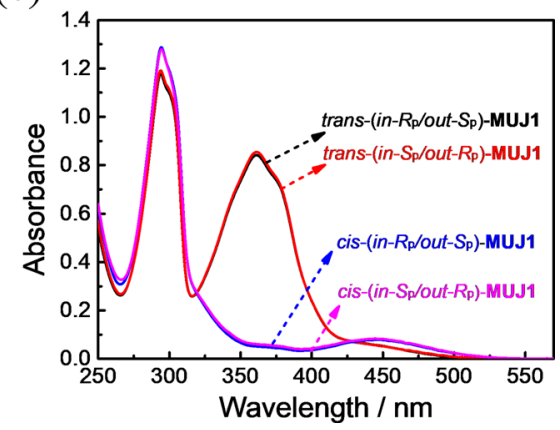

Fig. 3 Chiral HPLC spectra and spectrum analysis of the enantiomers of MUJ1. a Chiral HPLC trace of a mixture containing trans- and cis-MUJ1 (tetrahydrofuran:n-hexane $=1: 4$, detected at $319 \mathrm{~nm}$ ). b CD and (c) UV-vis spectra of enantiomeric pairs of trans-MUJ1 and cis-MUJ1 measured in the HPLC eluant (tetrahydrofuran:n-hexane $=1: 4$ ).

Each trans-MUJ is composed of a pair of isolatable planar chiral enantiomers (Fig. 1b) ${ }^{37,39}$. Successful chiral resolution of all three MUJs was obtained by chiral phase HPLC by using different mobile phases (Supplementary Figs. 35-37). As illustrated in Fig. 3a, four peaks, corresponding to two pairs of enantiomers of trans-MUJ1 and cis-MUJ1, were well separated with certain eluent compositions, in which the first and fourth fractions were trans-MUJ1, and the second and third fractions were cis-MUJ1. Photoirradiation of the mixture at $365 \mathrm{~nm}$ led to an inversion of the relative intensities of the peaks $a / d$ and $b / c$ (Supplementary Fig. S42).

Circular dichroism (CD) spectra of isolated fractions were measured in the eluate (tetrahydrofuran: $n$-hexane $=1: 4$ ) at room temperature. The four fractions of MUJ1 gave two sets of UV-vis spectra (Fig. 3c) and two pairs of mirror images in the CD spectra (Fig. 3b), in agreement with two pairs of enantiomers. The firstand third-eluted fractions of MUJ1 showed positive CD extrema $\left(\mathrm{CD}_{\mathrm{ex}}\right)$, and the second- and fourth-eluted fractions gave negative $\mathrm{CD}_{\text {ex }}$ (Fig. 3b). We have demonstrated that the negative $\mathrm{CD}_{\mathrm{ex}} \mathrm{ca}$. $310 \mathrm{~nm}$ corresponds to the $S p(S p, S p, S p, S p, S p, S p)$ configuration of $\mathrm{P}[6]$ derivatives ${ }^{30,35}$, and theoretical calculations confirmed that the $S p$ configuration of $\mathrm{P}[6]$ shows a negative $\mathrm{CD}_{\text {ex }}$ (Supplementary Fig. 157). However, since the azobenzene side ring could be either self-included (in conformer, Fig. 1b) or selfexcluded (out conformer), the chirality and $\mathrm{CD}$ response of the
MUJs should critically depend on the conformers they adopt, and therefore, these $\mathrm{CD}$ spectral results did not immediately allow for assignment of the absolute configurations of these isomers.

Absolute conformation of MUJs. Competitive complexation experiments of the MUJs with G1 or G2 were performed to clarify the absolute configurations of the MUJs (Fig. 4a-c). In chloroform, the weak negative $\mathrm{CD}_{\text {ex }}$ of the MUJ1 1st fraction (Fig. 4a) was enhanced upon increasing the concentration of G1. This suggested that pushing out of the side ring by complexation of G1 led to the $S_{\mathrm{p}}$ conformation of the pillar[6]arene core. The 1st fraction of MUJ1 (Fig. 3a) was thus assigned to be trans-(in$R_{\mathrm{p}} /$ out $\left.-S_{\mathrm{p}}\right)-\mathrm{MUJ1}$, and the 4 th fraction was trans- $\left(\right.$ in $-S_{\mathrm{p}} /$ out $\left.-R_{\mathrm{p}}\right)-$ MUJ1. Photoisomerization of trans- $\left(\right.$ in- $R_{\mathrm{p}} /$ out $\left.-S_{\mathrm{p}}\right)-$ MUJ1 and trans- $\left(\right.$ in $-S_{\mathrm{p}} /$ out $\left.-R_{\mathrm{p}}\right)-\mathrm{MUJ1}$ led to the 2 nd and 3rd fractions, respectively, indicating that the 2 nd fraction is cis- $\left(\right.$ in $-R_{\mathrm{p}} /$ out $\left.-S_{\mathrm{p}}\right)$ MUJ1 and that the 3 rd fraction is cis- $\left(\right.$ in $-S_{\mathrm{p}} /$ out $\left.-R_{\mathrm{p}}\right)-$ MUJ1 (Supplementary Fig. 38). The absolute configuration of each fraction of MUJ3 was assigned by the same procedure (Fig. 4c). G1 is too bulky for the cavity of pillar[5]arene, and the absolute configuration of fractions of MUJ2 was assigned based on the complexation experiment with G2 (Fig. 4b). However, little CD spectral change could be observed with trans-(in- $R_{\mathrm{p}} /$ out $-S_{\mathrm{p}}$ )MUJ2, even after adding a large excess amount of G2, suggesting 


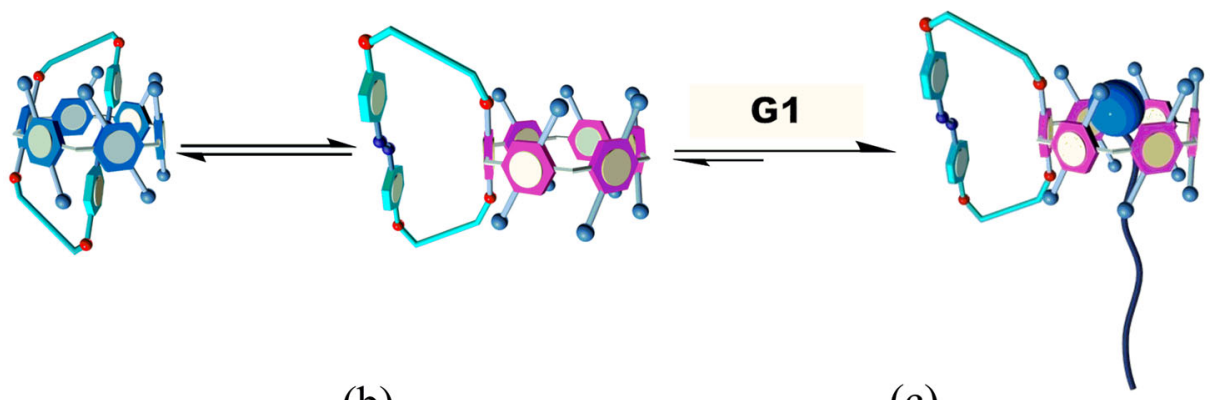

(a)

(d)
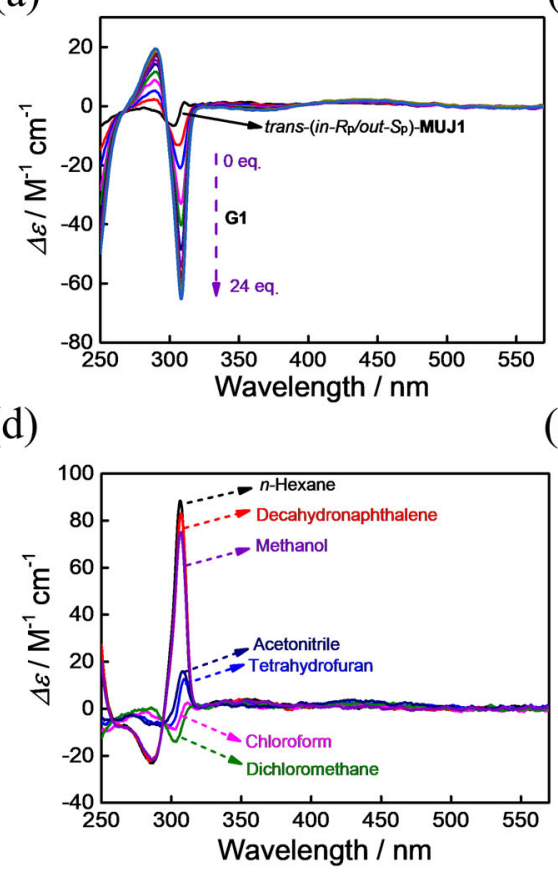

(b)

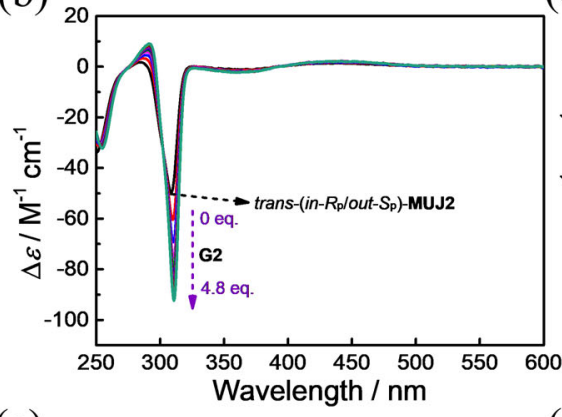

(e)

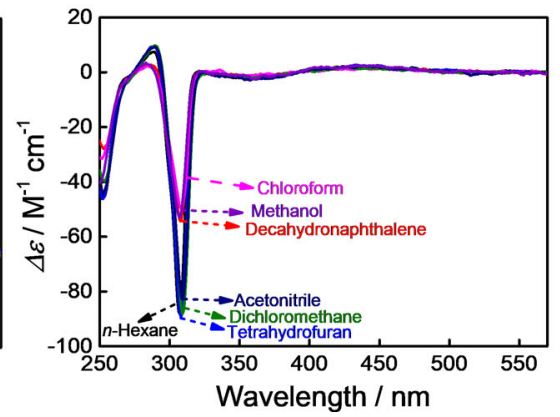

(c)

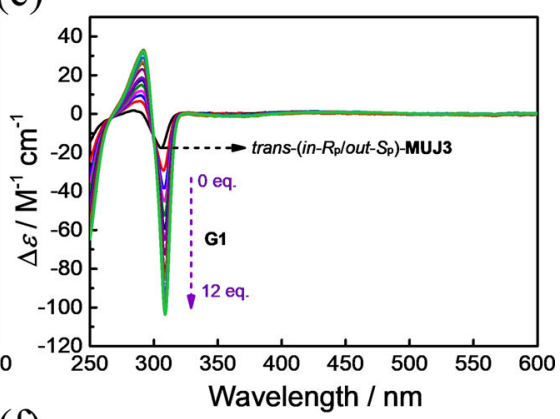

(f)

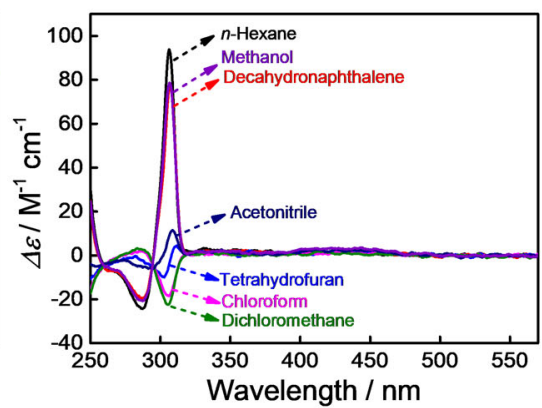

Fig. 4 Solvent effects and competitive complexation of MUJs. CD spectra of trans-(in- $R_{p} /$ out $\left.-S_{p}\right)$-MUJ1 (a) upon increasing the concentration of $\mathbf{G 1}$ in chloroform and (d) in different solvents; $C D$ spectra of trans-(in- $R_{\mathrm{p}} /$ out- $S_{\mathrm{p}}$ )-MUJ2 (b) with titrations of $\mathbf{G} \mathbf{2}$ in chloroform and (e) in different solvents; $C D$ spectra of trans-(in- $R_{\mathrm{p}} /$ out- $S_{\mathrm{p}}$ )-MUJ3 (c) upon increasing the concentration of $\mathbf{G 1}$ in chloroform at $25{ }^{\circ} \mathrm{C}$ and (f) in different solvents.

that trans-(in- $R_{\mathrm{p}} /$ out $\left.-S_{\mathrm{p}}\right)$-MUJ2 almost completely adopts the out conformer (Supplementary Fig. 44). Based on the titration experiments (Supplementary Figs. 43, 45 and 47) the association constants $K_{\text {assoc }}$ of $\mathbf{G 1}$ were determined as $(4.9 \pm 0.2) \times 10^{3} \mathrm{M}^{-1}$ for MUJ1 and $(7.5 \pm 0.4) \times 10^{3} \mathrm{M}^{-1}$ for MUJ3, respectively, and G2 showed a $K_{a s s o c}$ of $(8.0 \pm 0.8) \times 10^{4} \mathrm{M}^{-1}$ for MUJ2.

The CD spectra of the trans- $\left(\right.$ in $-R_{\mathrm{p}} /$ out $\left.-S_{\mathrm{p}}\right)$-MUJs were measured in various solvents (Fig. $4 \mathrm{~d}-\mathrm{f}$ ), including methanol, acetonitrile, dichloromethane, chloroform, tetrahydrofuran, decahydronaphthalene, and $n$-hexane. As illustrated in Fig. $4 \mathrm{~d}$, the CD spectra of trans- $\left(\right.$ in $-R_{\mathrm{p}} /$ out- $\left.S_{\mathrm{p}}\right)$-MUJ1 at room temperature were highly solvent-dependent: $\mathrm{CD}_{\mathrm{ex}}$ was strongly positive in the solvents $n$-hexane, decahydronaphthalene, and methanol and weakly positive in acetonitrile or tetrahydrofuran, indicating that a self-included (in) conformation dominated in all these solvents. However, $\mathrm{CD}_{\mathrm{ex}}$ was negative in chloroform or dichloromethane, suggesting that a self-excluded (out) conformer was adopted in these two solvents. The solvent-dependent CD spectra of trans$\left(\right.$ in $-R_{\mathrm{p}} /$ out $\left.-S_{\mathrm{p}}\right)$-MUJ3 were similar to those of trans- $\left(\right.$ in $-R_{\mathrm{p}} /$ out$S_{\mathrm{p}}$ )-MUJ1 (Fig. 4f). Such solvent effects reflect a delicate interaction balance between the in and out conformers, which involves binding of the solvent molecule(s), self-complexation of the side ring by the pillar[ $n]$ arene cavity, and solvation of the side ring. In particular, different solvation of the side ring and complexation of solvents by the pillar[ $n]$ arene cavity should be responsible for this distinct solvent dependence ${ }^{35}$. The strong positive $\mathrm{CD}_{\mathrm{ex}}$ observed in $n$-hexane, decahydronaphthalene and methanol should be ascribed to the unfavorable solvation effects of these solvents for the fused azobenzene moiety of MUJ1 and MUJ3, which facilitated a shift of the in-out equilibrium to form self-complexation conformers. In contrast, the P[5]-based trans(in- $R_{\mathrm{p}} /$ out $-S_{\mathrm{p}}$ )-MUJ2 always presented strong negative $\mathrm{CD}_{310}$ in all solvents examined, implying that the smaller cavity of $\mathrm{P}[5]$ hardly accommodated the azobenzene block of MUJ2, and the out conformation dominated in different solvents ${ }^{40}$. Furthermore, CD signals of trans-(in- $R_{\mathrm{p}} /$ out $\left.-S_{\mathrm{p}}\right)$-MUJ2 in acetonitrile, dichloromethane, and $n$-hexane were stronger than in chloroform, decahydronaphthalene, and methanol, presumably due to the strong complexation of the formers with pillar[5] arene which fixes the orientation of each subunit.

Light-driven chirality switching of MUJs. Trans- $\left(\right.$ in $-R_{\mathrm{p}} /$ out $\left.-S_{\mathrm{p}}\right)$ MUJ1 showed a strong positive $\mathrm{CD}_{\mathrm{ex}}$ in $n$-hexane at $20^{\circ} \mathrm{C}$ due to the self-included in- $R_{\mathrm{p}}$ conformation. Photoirradiation of MUJ1 at $365 \mathrm{~nm}$ led to a significant decrease in the absorption peak at $365 \mathrm{~nm}$ and an increase in the long-wavelength range absorption. Such absorption changes can commonly be used as readouts of azobenzene-based molecular switches. Interestingly, the strong positive $\mathrm{CD}_{\mathrm{ex}}$ of trans-(in- $R_{\mathrm{p}} /$ out $-\mathrm{S}_{\mathrm{p}}$ )-MUJ1 decreased and inverted to a strong negative CD signal upon irradiation at $365 \mathrm{~nm}$, and the weak CD signal peak at $435 \mathrm{~nm}$ significantly increased. Moreover, the original $\mathrm{CD}$ spectrum could be recovered by photoirradiation 
at $510 \mathrm{~nm}$, at which the ratio of the absorption spectrum of cisisomer divided by that of trans-isomer reaches the maximum (Fig. 6a and Supplementary Figs. 54-67). No fatigue was observed for the light-driven planar chirality switching between trans-in$\left(R_{\mathrm{p}}\right)$-MUJ1 and cis-out- $\left(S_{\mathrm{p}}\right)$-MUJ1 after several tens of cycles (Fig. 6b).

Such a CD spectral change indicated a switching from trans-in$\left(R_{\mathrm{p}}\right)$-MUJ1 to cis-out- $\left(S_{\mathrm{p}}\right)$-MUJ1, demonstrating that the planar chirality of MUJ1 can be switched by changing the irradiation wavelength. Such a photoinduced CD spectral change provides a powerful chiroptical switch that allows for distinctively determining on/off based on the positive/negative sign of $\mathrm{CD}_{\mathrm{ex}}$ rather than on the intensity, as commonly encountered in absorption and emission spectroscopic techniques. The rolling out of the side ring upon the trans to cis isomerization seems reasonable because the cis-azobenzene block became too bulky to be accommodated in the cavity of $\mathrm{P}[6]$, i.e., the trans-azobenzene block preferred to be included in the cavity, while it was excluded upon conversion to the cis-configuration (Fig. 5). Alternating photoirradiation of trans- $\left(\right.$ in- $R_{\mathrm{p}} /$ out $\left.-S_{\mathrm{p}}\right)-\mathbf{M U J 1}$ at $365 \mathrm{~nm}$ and $510 \mathrm{~nm}$ led to reversible switching of both the CD (anisotropy factor $g=\Delta \varepsilon / \varepsilon$, where $\varepsilon$ is the molar extinction coefficient at a particular wavelength, Fig. 6b) and UV-vis (Supplementary Figs. 56-67) spectra between their PSSs.

In contrast, trans-(in- $R_{\mathrm{p}} /$ out- $\left.S_{\mathrm{p}}\right)$-MUJ2 showed a strong negative $\mathrm{CD}_{\mathrm{ex}}$ in $n$-hexane at room temperature, suggesting that the trans-azobenzene moiety was located outside the P[5] cavity, which could be ascribed to the azobenzene moiety being too

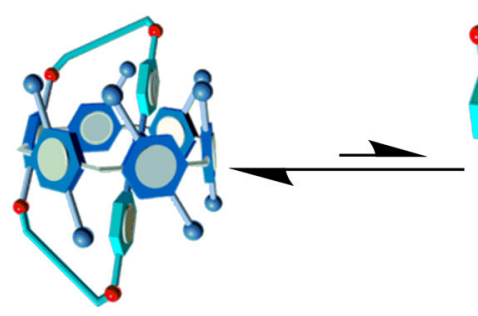

trans-in- $\left(R_{p}\right)$-MUJ1

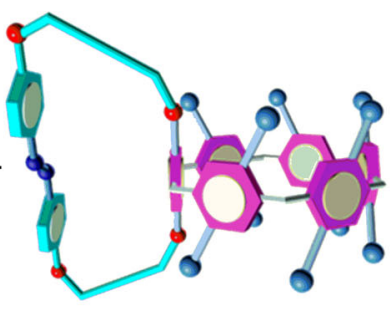

trans-out-( $\left.S_{p}\right)$-MUJ1

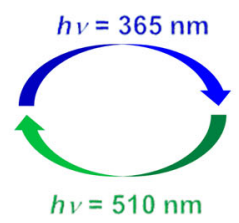

$h v=510 \mathrm{~nm}$

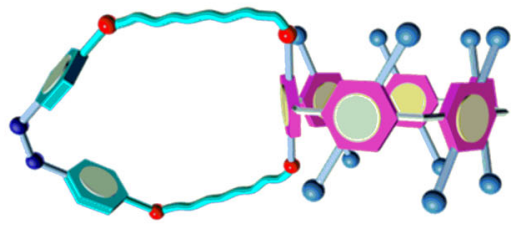

cis-out- $\left(S_{\rho}\right)$-MUJ1

Fig. 5 Light-driven chirality switching of (in- $\boldsymbol{R}_{\mathbf{p}} /$ out- $\left.\mathbf{S}_{\mathbf{p}}\right)-\mathbf{M U J 1}$. Suppositional mechanism of light-driven chirality switching of (in- $R_{\mathrm{p}} /$ out- $\left.\mathrm{S}_{\mathrm{p}}\right)-\mathbf{M U J 1}$

(a)
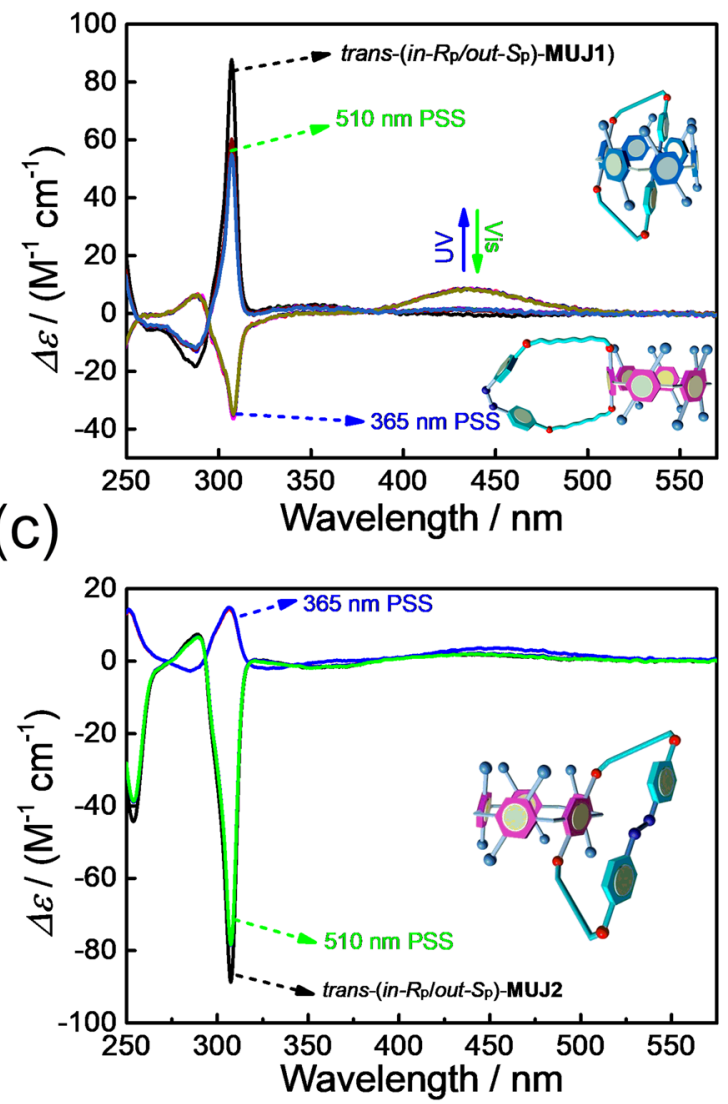

(b)

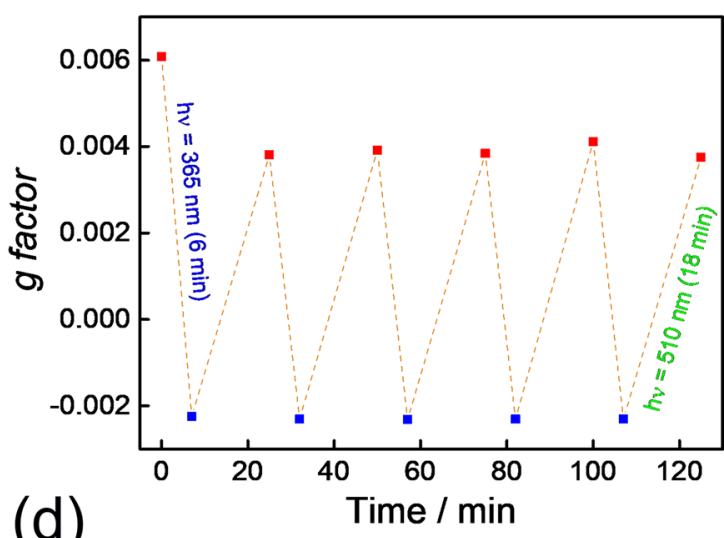

(d)

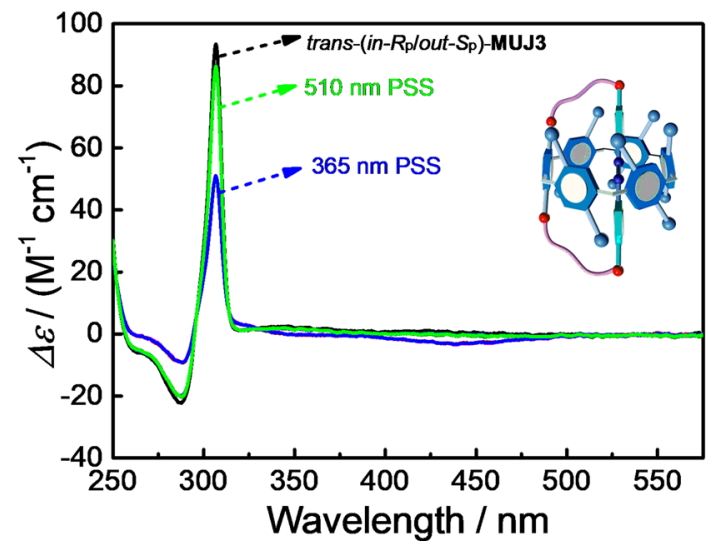

Fig. 6 CD spectral change of the enantiomers of MUJs upon selective wavelength photoirradiation. a CD spectra and (b) extremum changes ( 310 nm) of the anisotropy factor $(g)$ of trans-(in- $R_{p} /$ out- $S_{p}$ )-MUJ1 upon alternating irradiation at $365 \mathrm{~nm}$ (blue signet) and $510 \mathrm{~nm}$ (red signet, xenon light source) in $n$-hexane. CD spectra of (c) trans-(in- $R_{\mathrm{p}} /$ out- $S_{\mathrm{p}}$ )-MUJ2 and (d) trans-(in- $R_{\mathrm{p}} /$ out- $S_{\mathrm{p}}$ )-MUJ3 upon alternating irradiation at 365 and $510 \mathrm{~nm}$ in $n$-hexane. 
(a)

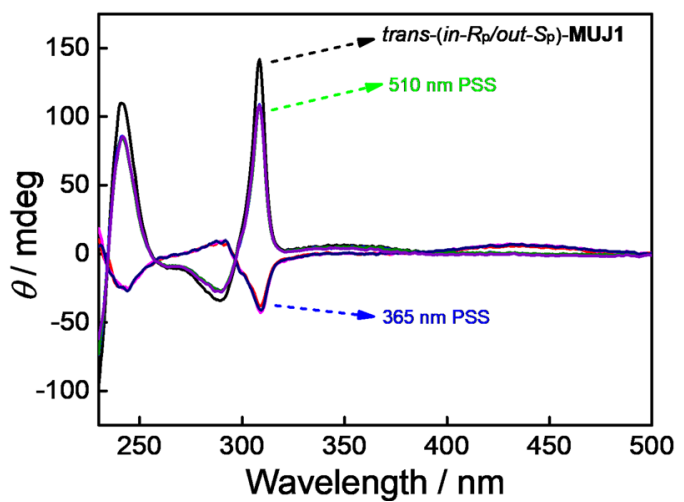

(b)

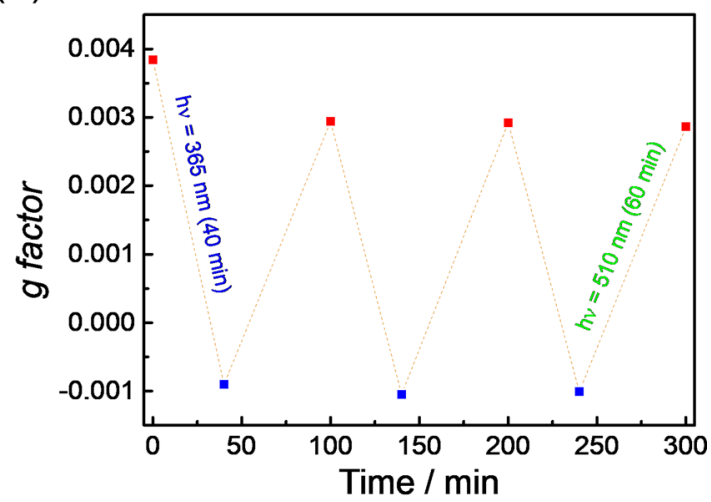

Fig. 7 Light-driven chirality switching of (in- $\boldsymbol{R}_{\mathbf{p}} / \mathbf{o u t}-\boldsymbol{S}_{\mathbf{p}}$ )-MUJ1 in the coating film. a CD spectra of the spin-coated film of trans-(in- $R_{\mathrm{p}} /$ out- $S_{\mathrm{p}}$ )-MUJ1 under alternating irradiation at 365 and $510 \mathrm{~nm}$ (xenon light source). b Extremum changes ( $310 \mathrm{~nm}$ ) of the anisotropy factor g of trans-(in- $R_{\mathrm{p}} /$ out- $S_{\mathrm{p}}$ )-MUJ1 upon alternating irradiation at $365 \mathrm{~nm}$ (blue signet) and $510 \mathrm{~nm}$ (red signet)

bulky to be comfortably accommodated by the $\mathrm{P}[5]$ cavity $^{41}$. Photoirradiation of trans- $\left(\right.$ in $-R_{\mathrm{p}} /$ out $\left.-S_{\mathrm{p}}\right)$-MUJ2 at $365 \mathrm{~nm}$ led to a significant decrease and almost inversion in $\mathrm{CD}_{\text {ex }}$ (Fig. $6 \mathrm{c}$ and Supplementary Figs. 68-69), suggesting that the out reference became weaker. Molecular model studies of trans-MUJ2 suggested that the side ring is stretched flat and is relatively rigid (Supplementary Fig. 84) due to the long strip structure of azobenzene, which prevents even partial inclusion of the side ring by the small pillar[5] arene cavity. However, the side ring in cisMUJ2 is much more flexible, which allows the triethylene glycol ether chain to be partially included in the $\mathrm{P}[5]$ cavity ${ }^{35}$ (Supplementary Figs. 85-86). Indeed, ${ }^{1} \mathrm{H}-\mathrm{NMR}$ spectra of MUJ2 recorded after $365 \mathrm{~nm}$ irradiation showed significantly upfield-shifted protons of the triethylene glycol ether chain, demonstrating self-inclusion of the fused glycol ether once transMUJ2 transformed to cis-MUJ2 (Supplementary Fig. 81). However, the cis-azobenzene block is too bulky for the $\mathrm{P}[5]$ cavity, which prevents complete chirality switching to the self-included conformer. The decreased negative $\mathrm{CD}_{\mathrm{ex}}$ caused by irradiation at $365 \mathrm{~nm}$ almost completely recovered to the initial state after irradiation at $510 \mathrm{~nm}$ (Fig. 6c and Supplementary Figs. 68-69).

Trans-(in- $R_{\mathrm{p}} /$ out- $\left.S_{\mathrm{p}}\right)$-MUJ3, which has a longer glycol ether chain and therefore a more flexible side ring than trans- $\left(i n-R_{\mathrm{p}} /\right.$ out- $S_{\mathrm{p}}$ )-MUJ1, also showed a strong positive $\mathrm{CD}_{\text {ex }}$ (Fig. $6 \mathrm{~d}$ ) in $n$ hexane, suggesting that the trans-azobenzene block of MUJ3 was self-included in the cavity. However, unlike the chiroptical inversion observed with MUJ1, the positive $\mathrm{CD}_{\mathrm{ex}}$ of MUJ3 was significantly decreased under irradiation at $365 \mathrm{~nm}$ and recovered with irradiation at $510 \mathrm{~nm}$. This phenomenon suggested that the side ring of MUJ3 was only partially excluded from the pillar[6] arene cavity when transformed to cis-azobenzene (Supplementary Figs. 72-75). We ascribed this to the more flexible side ring of MUJ3, which allows the glycol ether chain to be partially selfaccommodated in the $\mathrm{P}[6]$ cavity when the trans-azobenzene isomerizes to the cis-azobenzene, thus hampering the chirality switching of trans-(in- $R_{\mathrm{p}} /$ out- $\left.S_{\mathrm{p}}\right)$-MUJ3. The above result indicated that the steric effect was critically affected by the cavity size and the chain length in the present system, and appropriate size matching between the cavity and the side ring as well as the rigidity of the side rings is pivotal to the light-driven chirality switching behavior.

Interestingly, the light-driven chirality switching of trans-(in$R_{\mathrm{p}} /$ out- $S_{\mathrm{p}}$ )-MUJ1 could also be observed in the coating film. A film of trans-(in- $R_{\mathrm{p}} /$ out $-S_{\mathrm{p}}$ )-MUJ1 was prepared by spin-coating and then kept under vacuum to dry for $24 \mathrm{~h}$. The film showed the strong positive $\mathrm{CD}_{\mathrm{ex}}$ of trans-(in- $R_{\mathrm{p}} /$ out- $\left.S_{\mathrm{p}}\right)$-MUJ1, which changed to a strong negative $C_{\text {ex }}$ when irradiated at $365 \mathrm{~nm}$ but recovered to the positive $\mathrm{CD}_{\mathrm{ex}}$ under irradiation at $510 \mathrm{~nm}$ (Fig. 7a). The reversible light-driven chirality switching of trans(in- $R_{\mathrm{p}} /$ out- $S_{\mathrm{p}}$ )-MUJ1 could be repeated many times (Fig. $7 \mathrm{~b}$ ), indicating that the trans/cis isomerization of the azobenzene block can effectively trigger mechanical rolling in/out of the side ring ${ }^{42}$. Such light-driven planar chirality switching of the unimolecular system in the coating film makes MUJ1 a promising candidate for photoresponsive switch materials.

Overtemperature protection for light-driven chirality switching. Self-sensing and a feedback ability for temperature perturbances are critically important for intelligent microdevices, having potential applications in, for example, microenvironment temperature sensing and overtemperature protection of microcircuits. While inhibition of certain molecular chemical and physical processes by reducing the temperature is an oftenobserved phenomenon, inhibiting on/off molecular switching by increasing the temperature is challenging, particularly at the single-molecule level. Variable temperature CD (VT CD) spectra of trans-(in- $R_{\mathrm{p}} /$ out $\left.-S_{\mathrm{p}}\right)$-MUJs were examined in various solvents (Supplementary Figs. 87-107). Increasing the temperature led to a decrease in the $C_{\text {ex }}$ of trans- $\left(\right.$ in $-R_{\mathrm{p}} /$ out $\left.-S_{\mathrm{p}}\right)$-MUJ2 in all solvents examined (Fig. 8b and Supplementary Figs. 94-100), indicating that the out conformer trans-out- $\left(S_{\mathrm{p}}\right)$-MUJ2 was the main conformer in the temperature range examined. However, the CD spectra of trans- $\left(\right.$ in $-R_{\mathrm{p}} /$ out $\left.-S_{\mathrm{p}}\right)$-MUJ1 and trans- $\left(\right.$ in $-R_{\mathrm{p}} /$ out $\left.-S_{\mathrm{p}}\right)$ MUJ3 exhibited significant temperature dependence. For trans(in- $R_{\mathrm{p}} /$ out $-S_{\mathrm{p}}$ )-MUJ1, as an example, the negative $\mathrm{CD}_{\mathrm{ex}}$ in tetrahydrofuran at $50^{\circ} \mathrm{C}$ was reduced upon decreasing the temperature and finally inverted to give a positive $\mathrm{CD}_{\mathrm{ex}}$ at $5{ }^{\circ} \mathrm{C}$ (Fig. 8a), demonstrating a conformational transformation from trans-out- $\left(S_{\mathrm{p}}\right)$-MUJ1 to trans-in- $\left(R_{\mathrm{p}}\right)$-MUJ1. The UV-vis spectra of the trans-MUJs were hardly changed by the temperature variation (Supplementary Figs. 87-107), indicating no trans to cis isomerization of the azobenzene blocks with the temperature variation. Raising the temperature back to $50{ }^{\circ} \mathrm{C}$ led to a complete recovery of the initial CD spectrum of trans- $\left(\right.$ in $-R_{\mathrm{p}} /$ out $\left.-S_{\mathrm{p}}\right)$ MUJ1. The in conformer is favored at low temperatures, which seems reasonable because the in conformer should be stabilized by van der Waals contact, $\mathrm{C}-\mathrm{H} . . \mathrm{O}, \pi-\pi$, and dipole-dipole interactions between the $\mathrm{P}[6]$ cavity and the side ring but is entropy disfavored due to the loss of the motional and rotational 
(a)

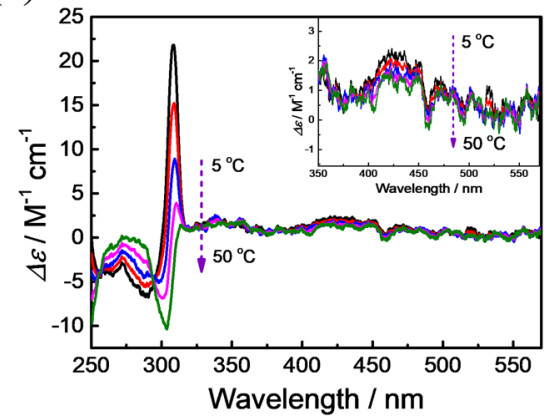

(b)

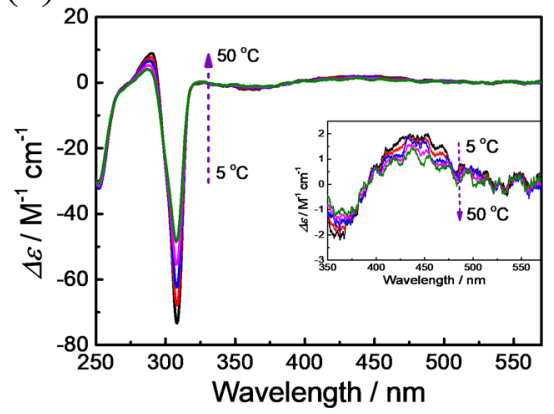

(c)

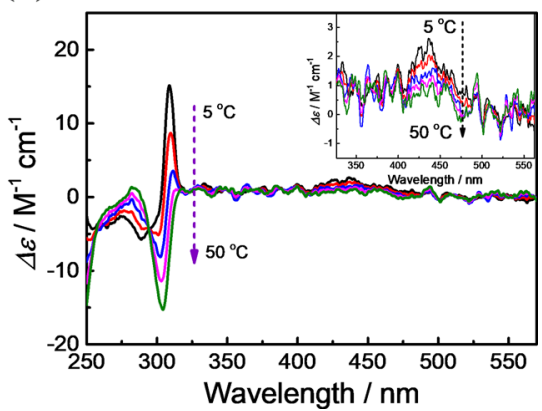

Fig. 8 Variable temperature CD spectra of the enantiomers of MUJs. VT CD spectra of (a) trans-(in- $R_{\mathrm{p}} /$ out- $\left.S_{\mathrm{p}}\right)-\mathbf{M U J 1}$, (b) trans-(in- $R_{\mathrm{p}} /$ out- $\left.S_{\mathrm{p}}\right)-\mathbf{M U J \mathbf { 2 }}$, and (c) trans-(in- $R_{\mathrm{p}} /$ out $-\mathrm{S}_{\mathrm{p}}$ )-MUJ3 in tetrahydrofuran at $5^{\circ} \mathrm{C}$ (black line), $15^{\circ} \mathrm{C}$ (red line), $25^{\circ} \mathrm{C}$ (blue line) $35^{\circ} \mathrm{C}$ (magenta line) and $50{ }^{\circ} \mathrm{C}$ (green line), respectively.
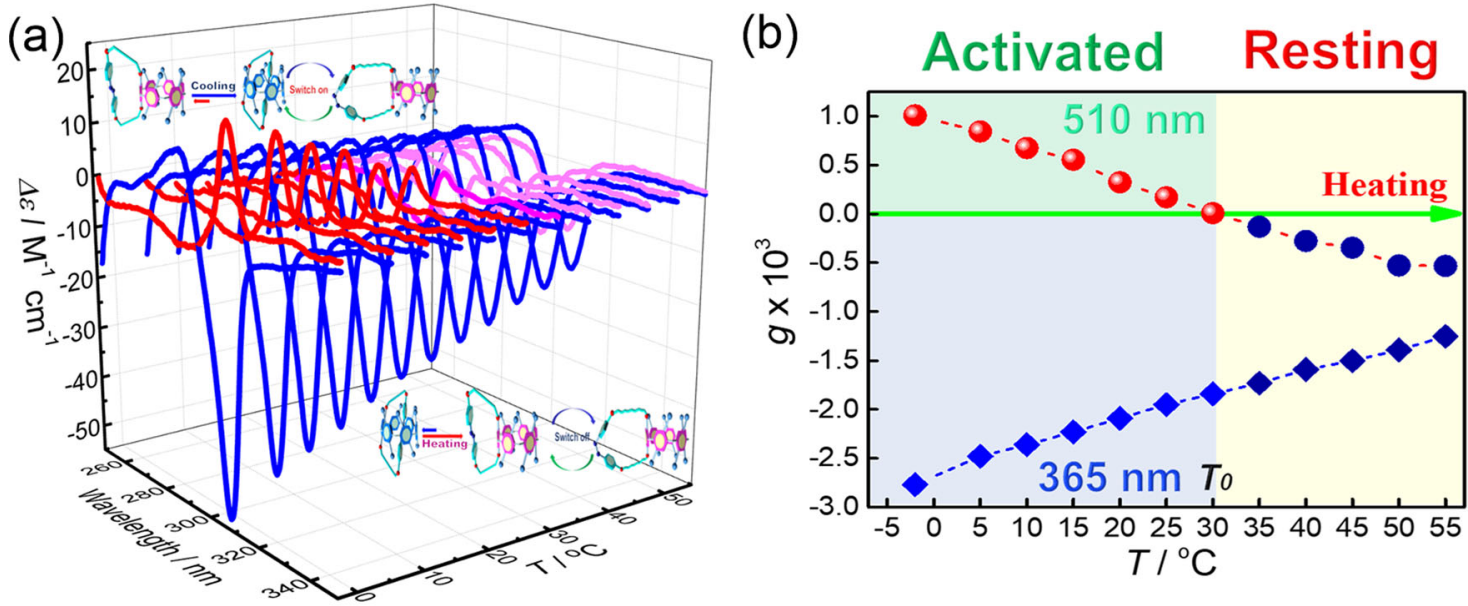

Fig. 9 Temperature regulation of the light-driven CD spectral inversion of enantiomeric MUJ1. a VT CD spectra of (in- $R_{p} /$ out- $\left.S_{p}\right)-M U J 1$ in the PSS at $365 \mathrm{~nm}$ (blue line) and $510 \mathrm{~nm}$ (red, magenta, and pink lines) in tetrahydrofuran. The CD spectra of (in- $R_{\mathrm{p}} /$ out- $S_{\mathrm{p}}$ )-MUJ1 with positive CD extrema (CD ex) at ca. $310 \mathrm{~nm}$ is shown as the red line, while that with negative $C D_{\text {ex }}$ is shown as the pink line and that between the two is shown as the magenta line. $\mathbf{b} V T$ anisotropy factor changes at $308 \mathrm{~nm}$ of (in- $R_{\mathrm{p}} /$ out- $S_{\mathrm{p}}$ )-MUJ1 in the PSS at $365 \mathrm{~nm}$ (diamond) and $510 \mathrm{~nm}$ (circle) in tetrahydrofuran. The blue signet presenting a negative $g$ factor and the red signet presenting a positive $g$ factor.

freedom of both the side ring and $\mathrm{P}[6]$ units. Temperature-driven chirality inversions were observed in tetrahydrofuran, chloroform, dichloromethane, and acetonitrile for MUJ1 (Fig. 8a and Supplementary Figs. 90-92) and in tetrahydrofuran and acetonitrile for MUJ3 (Fig. 8c and Supplementary Fig. 106). Such temperature-driven chiroptical switching demonstrated a delicate dynamic balance established by the significant entropy differences between the in and out conformers. It, therefore, provided a powerful tool to regulate the on/off switching of the MUJs and to implement sensing and feedback of microenvironmental temperature perturbances. Based on the VT CD spectral changes ${ }^{43}$, the entropy $(\Delta \Delta S)$ and enthalpy $(\Delta \Delta H)$ changes for the in/out conformational switching of MUJ1 in $\mathrm{CHCl}_{3}$ were estimated as $-16.5 \mathrm{~J} \mathrm{~mol}^{-1} \mathrm{~K}^{-1}$ and $-4.5 \mathrm{KJ} \mathrm{mol}^{-1}$, respectively. This demonstrates that the out-to-in conformational switching is enthalpically favorable but disfavored entropically.

The unique temperature and light dual-responsive property of MUJ1 endow it with the potential to serve as a temperatureresponsive molecular photoswitch. Irradiating trans $-\left(\right.$ in $-R_{\mathrm{p}} /$ out$S_{\mathrm{p}}$ )-MUJ1 at $-2{ }^{\circ} \mathrm{C}$ with light at 365 and $510 \mathrm{~nm}$ caused definite $\mathrm{CD}_{\mathrm{ex}}$ sign inversion (Fig. 9a), demonstrating the operation of light-driven switching. However, at $55^{\circ} \mathrm{C}$, trans- $\left(\right.$ in $-R_{\mathrm{p}} /$ out $\left.-S_{\mathrm{p}}\right)$ MUJ1 consistently showed a negative $\mathrm{CD}_{\mathrm{ex}}$ upon irradiation at both 365 and $510 \mathrm{~nm}$ (Fig. 9a), indicating silencing of the chirality inversion at high temperatures. Detailed investigation of the temperature-dependent photoswitching behavior (Fig. 9a) revealed a critical temperature $T_{0}$ of $30.3^{\circ} \mathrm{C}$ (Fig. 9b), below which $\mathrm{CD}_{\mathrm{ex}}$ exhibited positive/negative switching upon alternating photoirradiation with light at $365 \mathrm{~nm}$ and $510 \mathrm{~nm}$ and above which $\mathrm{CD}_{\mathrm{ex}}$ consistently exhibited a negative sign regardless of the irradiation wavelength employed. Therefore, the VT CD spectra of (in- $R_{\mathrm{p}} /$ out $\left.-S_{\mathrm{p}}\right)$-MUJ1 in the PSS at $365 \mathrm{~nm}$ were investigated (Supplementary Figs. 113-118) to gain insight into the temperature-regulated light-driven chirality switching. As shown in Fig. 9b, (in- $R_{\mathrm{p}} /$ out- $S_{\mathrm{p}}$ )-MUJ1 in the PSS at $365 \mathrm{~nm}$ presented a consistent negative $g$ factor in all of the examined temperature regime, while $\left(\right.$ in $-R_{\mathrm{p}} /$ out $\left.-S_{\mathrm{p}}\right)$-MUJ1 in the PSS at 510 $\mathrm{nm}$ presented a positive $g$ factor in the low-temperature regime $\left(T<30.3{ }^{\circ} \mathrm{C}\right)$ and a negative $g$ factor in the high-temperature regime $\left(T>30.3^{\circ} \mathrm{C}\right)$. This phenomenon indicated that temperature can serve as an effective external stimulus to regulate the light-driven chirality switching of (in- $R_{\mathrm{p}} /$ out- $\left.S_{\mathrm{p}}\right)$-MUJ1, which can be switched on by cooling and off by heating. As discussed above, the regulatory mechanism should be achieved by the synergistic effects between the two independent mechanisms, i.e., the temperature-regulated in-out equilibrium and the lightcontrolled photoisomerization of the azobenzene block (Supplementary Fig. 156). 


\section{Table 1 Critical temperature $\left(T_{0}\right)$ of $\left(\right.$ in- $R_{\mathrm{p}} /$ out- $\left.S_{\mathrm{p}}\right)-\mathrm{MUJ} 1$ in} different solvents.

\section{Solvent}

Tetrahydrofuran

$T_{0} /{ }^{\circ} \mathrm{C}(308 \mathrm{~nm})$

Acetonitrile

30.3

Chloroform

Acetonitrile:tetrahydrofuran $=1: 1$

Acetonitrile:tetrahydrofuran $=1: 19$

Acetonitrile:tetrahydrofuran $=19: 1$

47.6

29.7

8.8

31.0

34.0

Adjustable critical temperature of switching. Furthermore, the critical temperature $\left(T_{0}\right)$ for the regulation of the light-driven chirality switching can be adjusted by changing the solvents or solvent composition (Table 1). For example, the critical temperature $T_{0}$ was improved to $47.6^{\circ} \mathrm{C}$ in acetonitrile but decreased to $29.7^{\circ} \mathrm{C}$ in chloroform. Moreover, by using a mixture of acetonitrile and tetrahydrofuran as a solvent, $T_{0}$ could be conveniently adjusted from 8.8 to $34^{\circ} \mathrm{C}$ simply by changing the solvent composition. This unambiguously expands the application domains and improves the regulatory capabilities of the intelligent chirality switch.

\section{Discussion}

In summary, we demonstrated an intelligent molecular switch that shows a temperature- and photoresponsive on/off switch function. Such orthogonal control allows photoinduced rolling in/out and chiroptical switching to be prohibited when the temperature exceeds an upper limit, realizing overtemperature-protection intelligent molecular photoswitches. The upper-temperature limit could be conveniently adjusted by manipulating the solvent composition. This study realized the challenging hightemperature-gating effect at the molecular level and represents a prominent step forward for constructing an intelligent molecular machine/device capable of performing complex functions.

\section{Methods}

A Chiralpak IA column was used for chiral separation. The 365 and $510 \mathrm{~nm}$ light sources for photoresponsive $\mathrm{CD}$ spectral measurement were used with a grating spectrometer on a JASCO FP 8500 fluorescence spectrometer. A $365 \mathrm{~nm}$ LED spot curing system was used for photoresponsive ${ }^{1} \mathrm{H}-\mathrm{NMR}$ measurements. CD spectra were recorded as $\theta$ in millidegrees. The anisotropy factor $g$ was calculated using the equation $g=\Delta \varepsilon / \varepsilon$, where $\varepsilon$ is the molar extinction coefficient at a particular wavelength. The molar ellipticity was obtained by the formula $\Delta \varepsilon=\theta / 32980 \mathrm{cl}$, where $l$ is in $\mathrm{cm}$.

\section{Data availability}

The data are available upon reasonable request.

\section{Code availability}

The codes are available upon reasonable request.

Received: 8 January 2021; Accepted: 1 April 2021;

Published online: 10 May 2021

\section{References}

1. Kudernac, T. et al. Electrically driven directional motion of a four-wheeled molecule on a metal surface. Nature 479, 208-211 (2011).

2. García-López, V. et al. Molecular machines open cell membranes. Nature 548, 567-572 (2017).

3. Joachim, C., Gimzewski, J. K. \& Aviram, A. Electronics using hybridmolecular and mono-molecular devices. Nature 408, 541-548 (2000).
4. Coskun, A., Banaszak, M., Astumian, R. D., Stoddart, J. F. \& Grzybowski, B. A. Great expectations: can artificial molecular machines deliver on their promise? Chem. Soc. Rev. 41, 19-30 (2012).

5. Panman, M. R. et al. Operation mechanism of a molecular machine revealed using time-resolved vibrational spectroscopy. Science 328, 1255-1258 (2010).

6. Lee, C.-F. et al. Hybrid organic-inorganic rotaxanes and molecular shuttles. Nature 458, 314-318 (2009).

7. Qiu, Y. et al. A precise polyrotaxane synthesizer. Science 368, 1247-1253 (2020).

8. Zhu, K., Baggi, G. \& Loeb, S. J. Ring-through-ring molecular shuttling in a saturated [3] rotaxane. Nat. Chem. 10, 625-630 (2018).

9. Zhu, K., O'Keefe, C. A., Vukotic, V. N., Schurko, R. W. \& Loeb, S. J. A molecular shuttle that operates inside a metal-organic framework. Nat. Chem. 7, 514-519 (2015).

10. Koumura, N., Zijlstra, R. W., van, D. R. A., Harada, N. \& Feringa, B. L. Lightdriven monodirectional molecular rotor. Nature 401, 152-155 (1999).

11. van Delden, R. A. et al. Unidirectional molecular motor on a gold surface. Nature 437, 1337-1340 (2005).

12. Fletcher, S. P., Dumur, F., Pollard, M. M. \& Feringa, B. L. A reversible, unidirectional molecular rotary motor driven by chemical energy. Science $\mathbf{3 1 0}$, 80-82 (2005)

13. Stacko, P. et al. Locked synchronous rotor motion in a molecular motor. Science 356, 964-968 (2017).

14. Cheng, C. et al. An artificial molecular pump. Nat. Nanotechnol. 10, 547-553 (2015).

15. Qiu, Y. et al. A molecular dual pump. J. Am. Chem. Soc. 141, 17472-17476 (2019).

16. Guo, Q.-H. et al. Artificial molecular pump operating in response to electricity and light. J. Am. Chem. Soc. 142, 14443-14449 (2020).

17. Badjic, J. D., Balzani, V., Credi, A., Silvi, S. \& Stoddart, J. F. A molecular elevator. Science 303, 1845-1849 (2004).

18. Meng, Z. et al. Stepwise motion in a multivalent [2](3)catenane. J. Am. Chem. Soc. 137, 9739-9745 (2015)

19. Sun, J. et al. An electrochromic tristable molecular switch. J. Am. Chem. Soc. 137, 13484-13487 (2015)

20. Adam, A. \& Haberhauer, G. Switching process consisting of three isomeric states of an azobenzene unit. J. Am. Chem. Soc. 139, 9708-9713 (2017).

21. Borovkov, V. V., Hembury, G. A. \& Inoue, Y. The origin of solvent-controlled supramolecular chirality switching in a bis(zinc porphyrin) system. Angew. Chem. Int. Ed. 42, 5310-5314 (2003).

22. Van Zee, N. J. et al. Potential enthalpic energy of water in oils exploited to control supramolecular structure. Nature 558, 100-103 (2018).

23. Zhao, D., van Leeuwen, T., Cheng, J. \& Feringa, B. L. Dynamic control of chirality and self-assembly of double-stranded helicates with light. Nat. Chem. 9, 250-256 (2017)

24. de Jong, J. J. D., Lucas, L. N., Kellogg, R. M., van Esch, J. H. \& Feringa, B. L. Reversible optical transcription of supramolecular chirality into molecular chirality. Science 304, 278-281 (2004).

25. Zheng, Z.-g et al. Three-dimensional control of the helical axis of a chiral nematic liquid crystal by light. Nature 531, 352-356 (2016).

26. Koçer, A., Walko, M., Meijberg, W. \& Feringa, B. L. A light-actuated nanovalve derived from a channel protein. Science 309, 755-758 (2005).

27. Goujon, A. et al. Controlled sol-gel transitions by actuating molecular machine based supramolecular polymers. J. Am. Chem. Soc. 139, 4923-4928 (2017).

28. Bissell, R. A., Córdova, E., Kaifer, A. E. \& Stoddart, J. F. A chemically and electrochemically switchable molecular shuttle. Nature 369, 133-137 (1994).

29. Zahn, S. \& Canary, J. W. Electron-induced inversion of helical chirality in copper complexes of N,N-dialkylmethionines. Science 288, 1404-1407 (2000).

30. Xiao, C. et al. Redox-triggered chirality switching and guest-capture/release with a pillar[6]arene-based molecular universal joint. Angew. Chem. Int. Ed. 59, 8094-8098 (2020).

31. Spruell, J. M. et al. Highly stable tetrathiafulvalene radical dimers in [3] catenanes. Nat. Chem. 2, 870-879 (2010).

32. Biagini, C. \& Di Stefano, S. Abiotic chemical fuels for the operation of molecular machines. Angew. Chem. Int. Ed. 59, 8344-8354 (2020).

33. Fletcher, S. P., Dumur, F., Pollard, M. M. \& Feringa, B. L. A reversible, unidirectional molecular rotary motor driven by chemical energy. Science 310, 80-82 (2005).

34. Dulic, D., Kudernac, T., Puzys, A., Feringa, B. L. \& van Wees, B. J. Temperature gating of the ring-opening process in diarylethene molecular switches. Adv. Mater. 19, 2898-2902 (2007).

35. Yao, J. et al. Temperature-driven planar chirality switching of a pillar[5]arenebased molecular universal joint. Angew. Chem. Int. Ed. 56, 6869-6873 (2017).

36. Nagata, Y. et al. Holding of planar chirality of pillar[5]arene by kinetic trapping using host-guest interactions with achiral guest solvents. Chem. Commun. 56, 8424-8427 (2020). 
37. Ogoshi, T., Akutsu, T., Yamafuji, D., Aoki, T. \& Yamagishi, T.-A. Solvent- and achiral-guest-triggered chiral inversion in a planar chiral pseudo[1]catenane. Angew. Chem. Int. Ed. 52, 8111-8115 (2013).

38. Bandara, H. M. D. \& Burdette, S. C. Photoisomerization in different classes of azobenzene. Chem. Soc. Rev. 41, 1809-1825 (2012)

39. Li, S.-H., Zhang, H.-Y., Xu, X. \& Liu, Y. Mechanically selflocked chiral geminicatenanes. Nat. Commun. 6, 7590-7596 (2015)

40. Yu, G. et al. Pillar[6] arene-based photoresponsive host-guest complexation. J. Am. Chem. Soc. 134, 8711-8717 (2012).

41. Ogoshi, T., Yamafuji, D., Aoki, T. \& Yamagishi, T.-A. Photoreversible transformation between seconds and hours time-scales: threading of pillar[5] arene onto the azobenzene-end of a viologen derivative. J. Org. Chem. 76 9497-9503 (2011).

42. Zong, C. et al. Tuning and erasing surface wrinkles by reversible visible-lightinduced photoisomerization. Angew. Chem. Int. Ed. 55, 3931-3935 (2016).

43. Fan, C. et al. Precise manipulation of temperature-driven chirality switching of molecular universal joints through solvent mixing. Chem. Eur. J. 25, 12526-12537 (2019)

\section{Acknowledgements}

We acknowledge the support of this work by the National Natural Science Foundation of China (Nos. 92056116, 21871194, 21971169), National Key Research and Development Program of China (No. 2017YFA0505903), Key R \& D project of Science \& Technology Department of Sichuan Province (2019YJ0160, 2019YJ0090, and 2017SZ0021), CREST, JST, Japan (Grant No. JPMJCR2001), China Postdoctoral Science Foundation (No. 2019M653393), Comprehensive Training Platform of Specialized Laboratory, College of Chemistry and Prof. Peng Wu of Analytical \& Testing Center, Sichuan University.

\section{Author contributions}

C.Y. initiated the project. J.Y., W.W., and C.X. conceived and designed the experiments, analyzed the data, and prepared the manuscript, with input from all the authors. J.Y. conducted the chemical synthesis. D.S. and Z.Z. gave advice on CD and UV-Vis spectral analysis. T.M. conducted theoretical calculations of the CD spectra.

\section{Competing interests}

The authors declare no competing interests.

\section{Additional information}

Supplementary information The online version contains supplementary material available at https://doi.org/10.1038/s41467-021-22880-z.

Correspondence and requests for materials should be addressed to W.W. or C.Y.

Peer review information Nature Communications thanks Timothy Barendt and the other, anonymous, reviewer(s) for their contribution to the peer review of this work. Peer reviewer reports are available.

Reprints and permission information is available at http://www.nature.com/reprints

Publisher's note Springer Nature remains neutral with regard to jurisdictional claims in published maps and institutional affiliations.

\section{(c) (i)} appropriate credit to the original author(s) and the source, provide a link to the Creative Commons license, and indicate if changes were made. The images or other third party material in this article are included in the article's Creative Commons license, unless indicated otherwise in a credit line to the material. If material is not included in the article's Creative Commons license and your intended use is not permitted by statutory regulation or exceeds the permitted use, you will need to obtain permission directly from the copyright holder. To view a copy of this license, visit http://creativecommons.org/ licenses/by/4.0/.

(C) The Author(s) 2021 